Didáctica Geográfica n 20, 2019, pp. 193-212

ISSN: 0210-492-X

D.L: M-3736-2014

\title{
EL LENGUAJE CARTOGRÁFICO EN LOS LIBROS DE TEXTO DE GEOGRAFÍA DE SECUNDARIA EN BRASIL
}

Denis Richter ${ }^{1}$, Gabriel Martins Cavallini²

Recibido: 28/02/2019

Aceptado 15/09/2019

\section{RESUMEN:}

Este artículo es el resultado de una investigación que tuvo como objetivo analizar el lenguaje cartográfico presente en dos colecciones de libros de texto de geografía de secundaria en Brasil. Los mapas se analizaron en función de los diferentes tipos de representación cartográfica, así como las propuestas de cartografía escolar presentes en estos materiales didácticos. Como resultado, se observó que los mapas están restringidos a la cartografía euclidiana y contribuyen poco a la enseñanza de la geografía, teniendo como función principal solo localizar los fenómenos en el espacio.

\section{Palabras clave:}

Enseñanza de geografía, Mapas, Libros de texto.

\section{Abstract:}

This article is the result of an investigation that aimed to analyse the cartographic language present in two collections of high school geography textbooks in Brazil. The maps were analysed based on the different types of cartographic representation, as well

1 Instituto de Estudos Socioambientais (IESA). Universidade Federal de Goiás (UFG). Campus Samambaia, Itatiaia. Goiás. Brasil. drichter78@gmail.com

2 Instituto de Estudos Socioambientais (IESA). Universidade Federal de Goiás (UFG). Campus Samambaia, Itatiaia. Goiás. Brasil. cavallinigeografia@gmail.com 
as the proposals of school cartography present in these didactic materials. As a result, it was observed that the maps are restricted to Euclidean cartography and contribute little to the teaching of geography, having as its main function only to locate the phenomena in space.

\section{KEYWORDS:}

Geography teaching, Maps, Textbook.

\section{RÉSUMÉ:}

Cet article est le résultat d'une enquête visant à analyser le langage cartographique présent dans deux recueils de manuels de géographie de lycée au Brésil. Les cartes ont été analysées en fonction des différents types de représentation cartographique, ainsi que des propositions de cartographie scolaire présentées dans ces matériels didactiques. En conséquence, il a été observé que les cartes sont limitées à la cartographie euclidienne et contribuent peu à l'enseignement de la géographie, n'ayant pour fonction principale que de localiser les phénomènes dans l'espace.

\section{MoTS-CLÉ:}

Enseignement de la géographie, Cartes, Manuels scolaires.

\section{INTRODUCCIÓN}

La práctica de "mapear" o usar mapas de ubicación, espacialización de fenómenos y para definir territorios no es solo una característica de las sociedades contemporáneas. Por el contrario, esta práctica proviene de hace mucho tiempo. Este contexto hizo que el mapa fuese utilizado por diferentes áreas de conocimiento y no solo para el uso exclusivo de la ciencia geográfica. Así, su importancia se extiende a otros ámbitos como la biología, astronomía, sociología, historia, ingeniería, arquitectura, entre tantas otras profesiones. Pero no hay duda de que el geógrafo es quién tiene el gran potencial para construir, trabajar y comprehender los mapas en sus más diversas posibilidades.

Dicha relación entre el lenguaje cartográfico y la ciencia geográfica se manifiesta con mayor fuerza cuando realizamos una revisión histórica sobre la enseñanza de la geografía. En concreto, si se revisan los materiales didácticos que están diseñados para las actividades escolares de la enseñanza de la geografía. No es raro encontrar, en estas acciones didáctico-pedagógicas, el uso de los productos cartográficos más distintos para contribuir a la explicación del conocimiento geográfico.

Por lo tanto, en este artículo analizaremos cómo se presenta el lenguaje cartográfico en los libros de texto de geografía producidos para la escuela secundaria en Brasil. Esta 
investigación tendrá lugar en torno a dos colecciones aprobadas por el Plan Nacional de Libros Didácticos (PNLD) de 2018, a saber: a) Conexiones: Geografía general y Estudios de Brasil; b) Geografía - Espacio e identidad. Nuestra perspectiva es que este estudio nos permitirá un panorama, incluso sintético, de cómo se usa el lenguaje cartográfico en estos materiales, reconociendo su potencial y, al mismo tiempo, sus límites.

\section{CARTOGRAFía ESCOLAR EN EL AULA: ENTRE CONTENIDO Y LENGUAJE}

Pensar en el uso de la cartografía en el entorno escolar no es una tarea fácil. Hay varios factores que contribuyen e impiden el análisis y la comprensión del estudio de este contexto en el trabajo desarrollado en el aula. Debido a esto, en la segunda mitad de la década de los 70 en Brasil, la profesora Oliveira comenzó una serie de estudios sobre el uso que se hace de los mapas en la enseñanza las clases de geografía, basándose en su tesis titulada "Un estudio metodológico y cognitivo del mapa" (1978). En base a esta investigación, otros investigadores brasileños comenzaron a desarrollar trabajos en la misma perspectiva a lo largo de la década de 1980 hasta hoy, fortaleciendo los estudios sobre cartografía escolar (Almeida, 2007 y Richter, 2011).

Así, la cartografía comenzó a adquirir un papel más activo dentro de la ciencia geográfica en Brasil, dejando de ser simplemente un tema puntual abordado por algunos investigadores. De hecho, a partir de este momento comenzó a consolidarse como un área de investigación, alentando cada vez más la producción de obras que buscaban la construcción de teorías y metodologías sobre cartografía escolar. Su principal preocupación es entender cómo se lleva a cabo el proceso de enseñanza-aprendizaje a partir de los contenidos cartográficos y su lenguaje. Además, se busca la contribución de la cartografía a la escuela de geografía en relación con la enseñanza del/por el mapa y en la construcción del pensamiento espacial y geográfico.

Como señalamos en la introducción, los mapas son una parte constitutiva de la ciencia geográfica y, por lo tanto, de la enseñanza de la geografía. Pero después de todo, nos surge la siguiente pregunta ¿qué es un mapa? Podemos partir de la premisa, que al principio no existe una definición universal de lo que sería un mapa. Hay diferentes formas de construir un mapa, según su objeto, el fenómeno representado o su intencionalidad.

Según Martinelli (2017, p. 23), "la historia de la cartografía en el aula siguió la historia misma de la afirmación de este conocimiento, confirmando la enseñanza y el aprendizaje de la disciplina". Para comprender cómo la cartografía ha fomentado la enseñanza de la geografía, podemos hacer un breve viaje histórico, desde la perspectiva de las transformaciones que ocurrieron en la creación y uso de los mapas. 
El mapa existe desde las civilizaciones más antiguas, pasando por diferentes modelos de organización social y política. Según Harley (1991, p. 5) “el mapa auténtico más antiguo se hizo hace unos 6,000 años antes de Cristo y se descubrió en 1963 en el contexto de una excavación arqueológica en el centro-oeste de Turquía. Este mapa representaba el asentamiento neolítico del lugar donde fue encontrado (Çatal Höyük). Sin embargo, mapas como este no se consideraban verdaderamente como tal hasta hace poco, debido a la existencia de una perspectiva más positivista y una normativa de la cartografía que establece determinados estándares para la categorización y el reconocimiento de un mapa.

Sobre este largo y difícil proceso de aceptar otras formas de "mapeo", Harley (1991, p. 7) propone una definición más abierta para el mapa, siendo la "representación gráfica que facilita la comprensión espacial de objetos, conceptos, condiciones, procesos y hechos del mundo humano". Este argumento revela un cambio en los paradigmas y la búsqueda de expandir algo que ya estaba intrínsecamente universalizado. Esto demuestra que el mapa solo puede entenderse desde el contexto histórico y social en el cual se produjo y que, posteriormente, ofreció una base conceptual para su análisis.

En Brasil, estos cambios en la comprensión sobre el mapa contribuyeron a que se pensara más allá de sus normas técnicas o como un producto restringido al proceso de lectura de sus usuarios más que en el lenguaje cartográfico. Es decir, las prácticas relacionadas con su proceso de construcción se fortalecieron para mejorar el uso más consciente y crítico del mapa. En el currículo brasileño, desde los Parámetros Curriculares Nacionales (PCN) (BRASIL, 1998), se ha concedido una importancia significativa a los contenidos y al lenguaje cartográfico a lo largo de la educación obligatoria. Por tanto, esto debería ser visto como la comprensión de la relevancia de la cartografía para la formación intelectual y cognitiva de los estudiantes. Incluso con todo este progreso, Almeida (2013, p. 18) nos alerta sobre una preocupación:

A pesar de la importancia que este documento le dio a la cartografía fue un gran avance, merece la pena mencionar que se cometió el mismo error encontrado en los libros de texto, es decir, concentrar el tema en un solo punto del currículo, como si la representación pudiera separarse de los contenidos representados.

Estos elementos debemos entenderlos dentro de un proceso de enseñanzaaprendizaje, que empieza en Brasil en el sexto grado de la escuela primaria (11-12 años), cuando el alumnado posee una estructura cognitiva sobre los elementos básicos de la cartografía, y que se desarrolla hasta el último año de secundaria (17-18 años). A partir de esta última fase, el alumnado puede abordar temas más complejos y requieren 
un dominio de la lectura y la construcción del lenguaje cartográfico. Al referimos a los elementos básicos de la cartografía, destacamos los factores esenciales para la construcción de un mapa, que según Martinelli (2017) son: título, leyenda, orientación, coordenadas geográficas, escala, fuente y lenguaje en sí. Todos estos elementos se consideran contenidos de cartografía que deben trabajarse en el aula. Sin embargo, parece conveniente que los docentes desarrollen los contenidos cartográficos a lo largo de la formación escolar. En el currículo español, los contenidos cartográficos aparecen, esencialmente, en el tercer curso de educación primaria (García de la Vega, 2018). Para Fonseca (2013, p. 23):

La noción de imagen tiene un sentido amplio, casi sinónimo de representación y signo. Sin embargo, se puede tomar de manera más restringida, solo en el dominio visual. En este sentido, es un signo no verbal. Un mapa es una imagen visual compleja, cargada de signos no verbales que están estructurados como un sistema. Por lo tanto, el mapa es una pieza comunicativa, es lenguaje.

Richter (2017, p. 279) también destaca el hecho que el mapa se usa más allá del contenido al decir que "no basta con que el mapa simplemente esté presente, debe convertirse en un recurso que contribuya a las prácticas sociales de los individuos, desde el proceso de lectura hasta las propuestas para su construcción". Estos elementos presentes en el mapa caracterizan un lenguaje potente para la enseñanza de contenidos geográficos.

Cuando nos referimos a la cartografía como un lenguaje, también estamos hablando del uso de la llamada cartografía alternativa. Girardi (2012, p. 41) explica el término de "mapas alternativos", "en el contexto de los productos cartográficos, el significado dado por la palabra 'alternativo' como sustantivo tiende a ser más fuerte en nuestra imaginación, llevando el mapa a un dominio de combate social”. Por lo tanto, caracterizamos aquí los mapas alternativos como aquellos con un carácter más político y social, que tienen en cuenta los fenómenos a menudo en detrimento de la métrica euclidiana.

Se observa cada vez más el uso de diferentes modos de representación cartográfica, y estos se establecen en las perspectivas más diferentes, desde los mapas producidos a través de GoogleEarth o Google Maps, que se basan en su carácter científico-normativo, hasta las anamorfosis. Todas estas propuestas son formas diferentes de utilizar el lenguaje cartográfico en las clases de geografía.

Jacques Lévy (2008, p. 164) contribuye a esto diciendo que, 
Compare dos mapas electorales de los Estados Unidos, por ejemplo: el primer clásico y el segundo, que representan a los Estados Unidos con una superficie proporcional a su población. Esta confrontación muestra los efectos perversos de la representación euclidiana: al valorar las superficies vacías, es, bajo la apariencia de "precisión”, fundamentalmente falso tratar con fenómenos en los que es el número de hombres y no el inverso de su densidad lo que es la grandeza relevante.

Desde esta premisa, es importante señalar que no estamos aquí destacando una perspectiva del uso del lenguaje cartográfico en detrimento de otros. Se trata de potenciar los contenidos trabajados en dicho lenguaje, que en gran parte pueden usarse simultáneamente para mejorar la comprensión de los fenómenos, sus distorsiones y especificidades geográficas.

Por lo tanto, los contenidos geográficos y el lenguaje cartográfico se consideran como elementos inseparables en la construcción del conocimiento y de la enseñanza de geografía. Esto significa que el alumnado debe aprender contenidos geográficos y, específicamente, cartográficos. Incluso, si el único propósito de localizar fenómenos geográficos, apenas resulta significativo el aprendizaje de contenidos cartográficos.

Desde esta perspectiva, la cartografía escolar pretende contribuir a la construcción del pensamiento espacial y el pensamiento geográfico de los estudiantes. En suma, se busca asociar las prácticas escolares con el contenido y la cartografía para hacer que el proceso de enseñanza-aprendizaje sea cada vez más significativo.

\section{PENSAMIENTO COMPLEJO Y MAPAS EN LOS LIBROS DE TEXTO DE GEOGRAFÍA}

A partir de los contextos presentados hasta ahora, pudimos observar que los estudios que se centraron en la cartografía escolar se desarrollaron en línea con el uso diario de los mapas en el aula. Actualmente, en los dispositivos móviles se encuentran aplicaciones de geotecnologías, diferentes de la localización, o bien, como base para el desarrollo de otras actividades. Por lo tanto, podemos decir que esta situación de práctica social ha influido en el proceso de enseñanza y en las prácticas escolares que valoran el mapa como una herramienta relevante para la vida en sociedad. De hecho, los procesos derivados del pensamiento espacial proporcionan un aprendizaje para toda la vida (García de la Vega, 2019).

En los libros de texto de geografía, los mapas siempre han estado presentes. Sin embargo, en Brasil, a partir de la inserción de la obligación de usar el lenguaje cartográfico en el Programa Nacional de Libros Didácticos (PNLD), los mapas comenzaron a usarse de manera más amplia y sistemática en estos materiales escolares. Cabe destacar que, 
actualmente, en Brasil un libro de texto de geografía solo es aprobado por el Ministerio de Educación (a partir del PNLD) si presenta una propuesta de comunicación cartográfica asociada con los contenidos escolares.

En este sentido, varios investigadores empezaron a mirar cada vez más para comprender cómo los mapas podrían usarse en todo su potencial en la enseñanza de la geografía. Una de esas investigadoras, Simielli $(1986,1999)$, quien afirma la existencia de diferentes niveles de análisis cartográfico que los estudiantes deben comprender para leer un mapa. Y, añade esta autora que los mapas deberían permitirles leerlos a diferentes escalas en el contexto escolar.

Otra perspectiva con respecto a los mapas presentes en los libros de texto de geografía se refiere al nivel de complejidad de la cartografía, considerando el nivel cognitivo de los estudiantes. Según Duarte (2016), los mapas debieran adaptarse al desarrollo cognitivo de los estudiantes durante la etapa escolar. En secundaria, los mapas, presentes en el libro de texto del tercer año de educación básica en Brasil, muestran una mayor complejidad que la cartografía en los textos del primer año, atendiendo al desarrollo cognitivo.

En este sentido, observamos que la elaboración de los libros de texto de geografía ha carecido de interés en las investigaciones de las universidades brasileñas. A partir de la década de 1980, comenzó a publicarse un mayor número de materiales didácticos y, en consecuencia, generó el interés en investigaciones de este tipo. Según Santos (2003, p. 3):

Los años 80 están marcados por la mayor discusión y reflexión sobre el libro de texto de geografía, lo que resulta en la producción de libros de texto de mejor calidad, especialmente para la escuela secundaria, de numerosos títulos de paradigmas de geografía escritos por profesores de investigación de las universidades de São Paulo, de Río de Janeiro, Belo Horizonte, entre otros lugares.

A partir de esto, hay un punto de inflexión en la elaboración de los libros de texto de geografía en Brasil, que busca corregir los errores conceptuales y mejorar las propuestas metodológicas. La preocupación con estos elementos ahora se transmite de las entidades académicas que hasta entonces desarrollaron numerosas críticas a los materiales didácticos (Tonini et al. 2016).

Habiendo hecho esta pequeña retrospectiva sobre la consolidación de la investigación y las reformulaciones que involucran libros de texto, especialmente, de geografía, discutiremos los supuestos teóricos que guiaron el análisis de los mapas en nuestra investigación. Por lo tanto, buscamos comprender el lenguaje cartográfico en los libros de texto de geografía en secundaria a partir de los estudios de Simielli (1999) y Duarte (2016). 
Los libros de texto analizados en nuestra investigación presentaron una cantidad significativa de mapas. Para ello, conviene entender la presentación de este lenguaje en los libros. A fin de correlacionar esta presencia con los estudios teóricos del campo de la cartografía escolar. Con este fin, apoyamos nuestras discusiones y análisis en Simielli (1999, p. 97) en él presenta los niveles de actividad cartográfica:

Localización y análisis: mapas de análisis, distribución o cuadros de distribución, que analizan el fenómeno de forma aislada.

Correlación: permite la combinación de dos o más mapas de análisis.

Síntesis: muestra las relaciones entre varios mapas de análisis, presentados en un mapa-síntesis.

Simielli (1999) presenta los tres niveles de actividad cartográfica que, en su concepción, contribuyen al desarrollo cognitivo. Además, la lectura y análisis de los mapas fortalece las destrezas geográficas en los estudiantes. Estos niveles se desarrollarán a partir del proceso de aprendizaje de los estudiantes, que coincide con Duarte (2016). Este autor propone la necesidad de acompañar la etapa cognitiva de los estudiantes con las representaciones cartográficos

En cuanto a la propuesta de Simielli, conviene resaltar la diferencia entre los niveles cognitivos de los estudiantes de primaria y secundaria. En esta etapa de educación secundaria, los estudiantes alcanzan un mayor desarrollo cognitivo y, por lo tanto, pueden asimilar y problematizar más información que los estudiantes de primaria.

En los primeros años de primaria (6-11 años), según Simielli (1999, p. 97), el alumno tendrá contacto con los elementos de alfabetización cartográfica. Estos conocimientos básicos resultan necesarios para que el alumno pueda realizar análisis espaciales más complejos a lo largo de su formación escolar. Durante el sexto y noveno grado de la educación brasileña (11-15 años), el estudiante ya podrá desarrollar aspectos relacionados con la lectura y el análisis, y tal vez las correlaciones de cierta información. En la educación secundaria (15-18 años), el alumno alcanza el último nivel de actividad, pudiendo realizar lectura y análisis, correlación y síntesis de fenómenos geográficos presentes en una representación cartográfica. En esta etapa, el estudiante alcanza el nivel más elevado de las actividades relacionadas con el lenguaje cartográfico. 


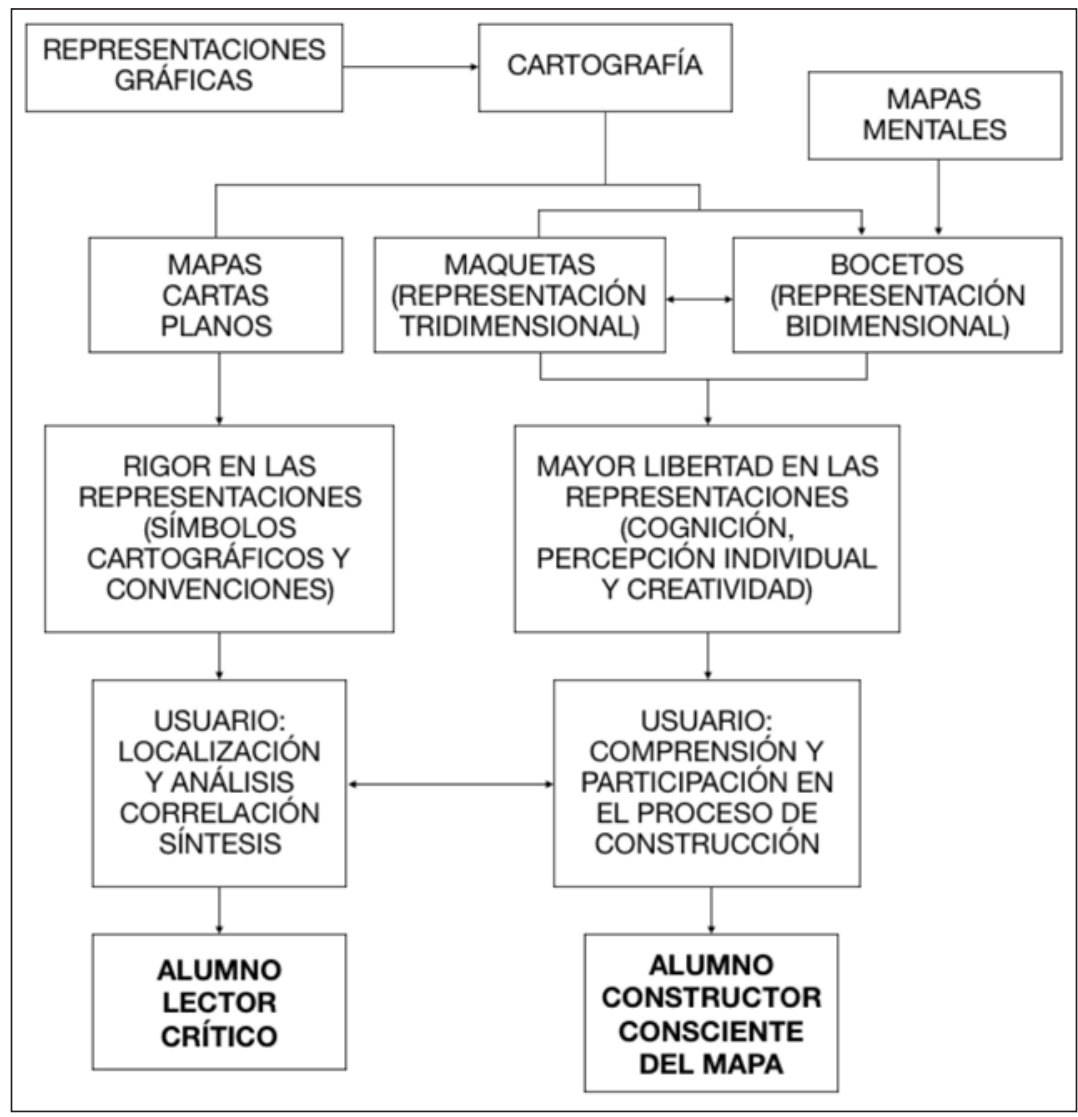

FIGURA N ${ }^{\circ}$ 1: La cartografía en la enseñanza de la geografía. Fuente: Simielli (1999, p. 101)

Por lo tanto, es un proceso continuo de construcción de conocimientos, vinculado al desarrollo cognitivo del alumno y su capacidad para interpretar los fenómenos y los contenidos geográficos. Así, Simielli (1999) presenta un mapa conceptual en el que organiza los procesos y elementos que componen la cartografía en el entorno escolar (Figura 1). Esta autora construye dos ejes distintos pero complementarios que conducen a dos perspectivas del alumno, el lector crítico y el constructor consciente del mapa.

De acuerdo con la Figura 1, en el primer eje el trabajo será con los productos cartográficos previamente elaborados, como mapas, cartas y planos. Por lo tanto, los elementos trabajados serán los que ya se han dado a los estudiantes y, a partir de este contexto, los niveles de actividad se emplearán a medida que avancen a los contenidos de los años siguientes. La expectativa de este proceso es la formación de un estudiante lector crítico, es decir, que tenga la capacidad de analizar, correlacionar y sintetizar la información presente en un mapa previamente preparado con conocimiento geográfico. 
En el segundo eje, las actividades desarrolladas contarán con la participación activa del alumno, mediante la realización de modelos, bocetos, mapas mentales y otros. Por lo tanto, deja de ser solo un lector y se convierte también en un constructor del mapa, de modo que los elementos trabajados pasarán por la técnica, pero también por la subjetividad, en el caso de los bocetos y los mapas mentales.

En este proceso, los niveles de actividad se consolidan en la construcción de representaciones, considerando que el alumno debe buscar información sobre el fenómeno que representará, localizará y, posteriormente, analizará. Este material puede correlacionarse con otros ya producidos, o bien, se realizarán. Y, finalmente, el alumnado puede construir una síntesis de estos contenidos trabajados.

Los niveles de actividad son importantes, porque a partir de ellos, a nuestro parecer, se lleva a cabo la construcción del conocimiento geográfico y la alfabetización cartográfica. Y en el caso de los libros de texto, permite al estudiante ser un lector crítico de mapas, extrapolando la función de solo ubicar los fenómenos.

Corroborando esta idea, Duarte (2016) presenta algunas preocupaciones, entre ellas que los mapas presentes en los libros de texto son solo para localización y que tampoco corresponden al nivel cognitivo de los estudiantes. Este investigador también destaca otros desafíos que están presentes al trabajar con mapas en la escuela:

- Uso reducido del mapa como recurso didáctico por muchos profesores de geografía.

- Abandonar, en particular, el Atlas Geográfico Escolar en los años posteriores al sexto grado de la escuela primaria.

- Un número extremadamente pequeño de preguntas relacionadas con la interpretación de mapas en pruebas y actividades pedagógicas en la disciplina de Geografía del segundo segmento de la escuela primaria y secundaria.

- Baja competencia en habilidades relacionadas con el uso e interpretación de representaciones cartográficas por parte de muchos estudiantes al final de la escuela secundaria.

- Bajo nivel de competencia en el campo del lenguaje cartográfico por una gran parte de los maestros en el primer segmento de la escuela primaria y una parte considerable de aquellos calificados para enseñar geografía en el segundo segmento de la escuela primaria y secundaria.

- Concentración de la enseñanza instrumental cartográfica en el sexto grado en la mayoría de los programas oficiales y colecciones de Geografía editados para el segundo segmento de la educación primaria.

- Uso extremadamente frecuente del mapa como ilustración y no como recurso de aprendizaje en la gran mayoría de los libros de texto básicos de geografía. 
- Testimonios de graduados de Geografía, matriculados en las asignaturas iniciales de formación docente, que no estaban calificados para trabajar con la Cartografía que debería enseñarse a los estudiantes de educación básica. (Duarte, 2016, p. 19-20)

Desde estos puntos, entendemos que la idea de usar el mapa todavía está excesivamente vinculada a la ilustración y no al desarrollo cognitivo, tal como lo enfatiza Simielli (1999), tomando como perspectiva la formación de los estudiantes como lectores críticos y constructores conscientes de mapa. Por lo tanto, es importante enfatizar que nuestro análisis se basa en el contexto de que los estudiantes de secundaria son alfabetizados cartográficos y, por lo tanto, tienen un mayor nivel de desarrollo cognitivo.

Por lo tanto, entendemos que los mapas en los libros de texto de geografía de secundaria deben corresponder a los niveles de desarrollo cognitivo de los estudiantes, y el maestro es responsable de la mediación de la construcción del conocimiento geográfico derivado del trabajo con el plan de estudios de materiales escolares y del currículo.

Para hacerlo, insertamos nuestra concepción en la idea de complejidad, es decir, que los mapas se vuelven más complejos a medida que aumenta el nivel cognitivo de los estudiantes. Por lo tanto, los mapas que se utilizarán para el trabajo de contenido con estudiantes de tercer año pueden, y con suerte, ser más complejos que los utilizados con estudiantes de primer año en esta misma fase de escolarización.

La teoría de la complejidad se puede encontrar desde diferentes perspectivas y por diferentes autores. En este artículo utilizamos la concepción de Morin (2000, p. 3539) que articula cuatro elementos para la construcción del conocimiento pertinente, que se presenta en funciónn de: el contexto, lo global, lo multidimensional y lo complejo. Así, para que la construcción del conocimiento sea significativa, es necesario vincular a nuestra investigación, mapas y contenidos geográficos, para que sea efectivo el trabajo desde estos niveles de expansión de complejidad.

En línea con la preocupación planteada por Duarte (2016), los mapas deben superar el estado de ser solo con fines ilustrativos y, como señala Simielli (1999), la ubicación y el análisis comprenden los niveles más simples de actividad. Como señaló Morin (2000), uno de los elementos para la formación del pensamiento complejo es el contexto, que se refiere a la idea de que "el conocimiento de la información o los datos aislados es insuficiente. Es necesario situar la información y los datos en su contexto para que adquieran significado" (Morin, 2000, p. 36). Por lo tanto, se espera que los mapas permitan al profesor, como mediador, y al estudiante, como constructor de conocimiento, transponer la acción solo de ilustración de fenómenos representados en los mapas. 


\section{Denis Richter y Gabriel Martins Cavallini}

Siguiendo el desarrollo cognitivo y avanzando en los años escolares, se espera que el estudiante pueda hacer correlaciones entre diferentes representaciones. En esta perspectiva, lo global tiene lugar porque:

Lo global es más que el contexto, es el conjunto de varias partes vinculadas a él de forma interretroactiva u organizativa. [...] El conjunto tiene calidad o propiedades que no se encuentran en las partes si están aisladas unas de otras, y ciertas cualidades o propiedades de las partes pueden verse inhibidas por las restricciones derivadas del conjunto. (Morin, 2000. p. 37)

La correlación de fenómenos geográficos permite una comprensión más amplia y compleja de los elementos cartográficos. A su vez, esto proporciona a los estudiantes desarrollar un pensamiento más complejo sobre su realidad, su vida diaria y, por lo tanto, su visión del mundo.

Ya lo multidimensional corrobora la pluralidad representativa de mapas en los libros de texto, ya que es posible mediante una colección de mapas presentar diferentes perspectivas de la misma realidad. Para Morin (2000, p. 38), la sociedad actual "incluye las dimensiones históricas, económicas, sociológicas, religiosas... pero las partes el uno del otro". La articulación que se puede realizar a través de más de un mapa, permite la comprensión de la pluralidad de aspectos sociales, físico-naturales, económicos, entre otros.

Todos estos elementos culminarán en el conocimiento pertinente, que, para Morin (2000, p. 38) es complejo, y en este sentido:

El conocimiento relevante debe enfrentar la complejidad. Complexus significa lo que ha sido entretejido, de hecho, hay complejidad cuando diferentes elementos son inseparables constitutivos del todo (como económico, político, sociológico, psicológico, afectivo, mitológico), y hay un tejido interdependiente, interactivo e interdependiente. retroactivo entre el objeto de conocimiento y su contexto, las partes y el todo, el todo y las partes, las partes entre sí.

En esta perspectiva, podemos correlacionar lo complejo con el proceso de síntesis, porque el estudiante usa las partes para componer el todo, y este todo, a su vez, se configura como el entrelazado de las partes, componiendo un pensamiento más complejo sobre el fenómeno retratado. En resumen, según Morin (2000, p. 38), "la complejidad es la unión entre la unidad y la multiplicidad", lo que para nosotros resulta en un estudiante alfabetizado cartográficamente, capaz de tejer razonamiento espacial y geográfico, además de ser capaz de articularlos de acuerdo con la construcción de pensamientos espaciales y geográficos.

Dicho esto, es importante enfatizar que la complejidad es esencial para la construcción del conocimiento geográfico, así como para el desarrollo cognitivo de los estudiantes. 
Por lo tanto, vislumbrar un lenguaje cartográfico complejo en los libros de texto le permite al maestro progresar mejor en la planificación de las clases y el trabajo con los contenidos, mientras que para el estudiante es significativo desde el punto de vista de la potencialidad de este lenguaje en el desarrollo del razonamiento y el aprendizaje.

\section{MAPEANDO EL LIBRO DE TEXTO DE GEOGRAFÍA DE SECUNDARIA: UN ANÁLISIS DEL LENGUAJE CARTOGRÁFICO}

Después de haber mantenido un diálogo sobre el campo teórico y metodológico de la enseñanza de la geografía, la cartografía escolar y el libro de texto, es hora de analizar los datos, que fueron recopilados desde una perspectiva cualitativa. Este método fue importante porque nos permitió usar una mirada más detallada y significativa de los números obtenidos, para superar una lectura dura y descontextualizada de los resultados.

Así, es importante enfatizar que los datos deben analizarse desde un contexto, que en este caso se refiere al hecho de que los libros de texto en Brasil se consideran documentos oficiales y, por lo tanto, se basan en pautas y análisis de verificación previa de un grupo de profesionales antes de llegar a las escuelas y ser utilizados por los estudiantes. Por otro lado, no señalamos en este documento los errores que pueden estar relacionados con la falla de los revisores en evaluar o elaborar, porque nuestro objetivo o propósito no es eso, sino discutir la no utilización en todo su potencial del lenguaje cartográfico ya existente en estos libros de texto.

Inicialmente, la selección de libros se basó en la lectura de las reseñas disponibles en la Guía del Plan Nacional de Libros de Texto (BRASIL, 2017), un documento mediante el cual se seleccionaron las seis colecciones que se analizarán en la investigación.

En este trabajo, los criterios principales de selección de los libros de texto, que son: a) la colección debe haber sido aprobada por el PNLD 2018; b) tener en la revisión provista por la Guía PNLD 2018 que la colección referida presenta un buen uso/tratamiento del lenguaje cartográfico; y c) tres de estas colecciones seleccionadas también deben haber sido aprobadas en el PNLD anterior de 2015. En este artículo presentamos datos que se refieren a dos colecciones, considerando que cada colección contiene tres libros, que se utilizan a lo largo de educación secundaria. Por tanto, esta investigación se analizan seis libros, esto es, tres libros de texto de cada colección ${ }^{3}$.

El instrumento utilizado para el análisis de las colecciones fue un formulario, mediante la plataforma Google. El formulario aparece elaborado por los investigadores a partir de los objetivos de este estudio. En este instrumento de análisis, además de verificar

3 A medida que la investigación continúa, se pretende finalizar el análisis de las seis colecciones en julio de 2020. 
las proposiciones de Simielli (1999) y Duarte (2016), también enumeramos elementos como: nombre de la colección, serie, número de página, título del mapa, corte espacial, tamaño del mapa, tipo de cartografía (euclidiana o no euclidiana).

Cada mapa se analizó independientemente de los libros que se refieren a los años de la escuela secundaria. Las respuestas se registraron completando el formulario en línea que alimentaba automáticamente una base de datos. A su vez, se iba generando una hoja de cálculo detallada de cada representación cartográfica, lo que permitió el análisis y la elaboración de tablas y gráficos.

El universo de esta investigación en este texto se refiere a los libros de texto de Geografía de dos colecciones de escuelas secundarias aprobadas en PNLD 2018, que se titulan: a) Conexiones: estudios de geografía general y Brasil; y b) Geografía: espacio e identidad. En el conjunto de estos libros se analizaron 367 mapas, excluyendo las actividades. Por lo tanto, investigamos solo las representaciones cartográficas que se insertaron en el cuerpo del texto, siguiendo los contenidos.

Este elevado número de mapas puede considerarse, a priori, un dato positivo para los investigadores en el campo de la cartografía escolar en Brasil. Siempre ha sido una de las preocupaciones presentadas con respecto a la escasa utilización de mapas en las clases de geografía en la escuela. Sin embargo, es necesario mostrar cautela, porque no es suficiente que el lenguaje cartográfico solo esté presente en los materiales. Es necesario que los mapas se articulen con las propuestas de los libros, la mediación del maestro y la posibilidad de análisis, lectura y construcción por parte del alumnado.

Por lo tanto, se trata de comprender el tipo de cartografía, ya que podemos enumerar una diversidad de cartografías. Así pueden ser, por ejemplo, la cartografía sistemática y temática, el boceto, la anamorfosis, el mapa mental y narrativo, entre tantas otras posibilidades. En vista de esto, uno de los puntos de este análisis tenía que ver con el tipo de cartografía que se encuentra en los libros. Con este fin, los dividimos en dos grandes categorías, que son: a) cartografíaa euclidiana; b) cartografía no euclidiana (Tabla 1).

\begin{tabular}{|c|c|c|c|c|c|c|}
\hline \multirow{2}{*}{$\begin{array}{c}\text { Tipo de } \\
\text { cartografía }\end{array}$} & \multicolumn{3}{|c|}{$\begin{array}{c}\text { Conexiones: estudios de geografía } \\
\text { general y Brasil }\end{array}$} & \multicolumn{3}{c|}{ Geografía: espacio e identidad } \\
\cline { 2 - 7 } & $1^{\circ}$ Año & $2^{\circ}$ Año & $3^{\circ}$ Año & $1^{\circ}$ Año & $2^{\circ}$ Año & $3^{\circ}$ Año \\
\hline $\begin{array}{c}\text { Cartografía } \\
\text { euclidiana }\end{array}$ & 55 & 53 & 58 & 66 & 74 & 58 \\
\hline $\begin{array}{c}\text { Cartografía } \\
\text { no euclidiana }\end{array}$ & 0 & 0 & 0 & 2 & 0 & 1 \\
\hline
\end{tabular}

TABLA $^{0} 1$ : Tipos de cartografía presentes en las colecciones** por número de aparición. Fuente: Elaboración propia (2019) 
Al analizar esta tabla 1, observamos un hecho, que no resultó sorprendente. Encontramos una gran cantidad de representaciones de la cartografía euclidiana, dedicada, principalmente, a mapas sistemáticos y temáticos. Este contexto puede explicarse a partir de los estándares requeridos por el Ministerio de Educación (a partir del PNLD) para aprobar un libro de texto. En este documento se enumeran algunos criterios técnicos para el uso, inserción y validación de mapas en los materiales didácticos. Entre estos, se presenta la cuestión de la morfología del mapa que debe contener: título, leyenda, escala, orientación y fuente.

Este factor dificulta, por lo tanto, la inserción de la cartografía no euclidiana, o como lo destaca la cartografía subversiva de Seemann (2012), ya que este tipo de representaciónn suprime uno o más elementos cartográficos. Por lo tanto, enfatizamos que nuestra postura no consiste en abogar por el uso de un solo modelo de cartografía sobre otro. Se señala que ambos pueden y deben emplearse de manera integrada para trabajar los contenidos geográficos.

Las potencialidades de estas cartografías se presentan en diferentes perspectivas y son, sobre todo, complementarias. Al mismo tiempo se recurre a mapas sistemáticos para trabajar con contenidos cartográficos y geográficos, como contornos e índices de lluvia, que respaldan el análisis de ciertos aspectos. Los aspectos físico-naturales, por otro lado, buscan en los mapas de anamorfosis representaciones de la densidad de población y la distribución del ingreso, por ejemplo, que mejoran el análisis de este fenómeno que se representará en el mapa. Por lo tanto, entendemos que la medición no siempre es el elemento clave para analizar el espacio geográfico, todo depende del objetivo de enseñanza.

Además de este contexto del tipo de cartografía presente en los libros de texto, nuestro análisis de mapas también se basó en las perspectivas de los niveles de actividad cartográfica propuestos por Simielli (1999), Además, también aparece el desajuste entre el nivel cognitivo de los estudiantes y la complejidad del mapa presente en los libros de texto, idea presentada por Duarte (2016).

Por lo tanto, es posible observar que estos dos criterios de análisis tratan, desde la perspectiva de Simielli (1999), si los mapas presentes en los libros de texto permiten a los estudiantes desarrollar los tres niveles de análisis, en los que la ubicación y el análisis se configuran como más simple, posteriormente la correlación como nivel intermedio y la síntesis siendo el nivel más alto. Mientras que para Duarte (2016) su atención se centra en el análisis de cómo se usaron los mapas en los libros de texto de geografía para los últimos años de la escuela primaria, con la perspectiva de utilizar el lenguaje cartográfico más allá de la localización y valorar el desarrollo del pensamiento más complejo de estudiantes de leer y analizar mapas. Para esto, este autor también establece tres niveles de complejidad para analizar el lenguaje cartográfico, que son bajo, medio y alto. 
En resumen, ambos enfoques resaltan que hay un proceso de lectura de mapas que debe desarrollarse en las clases de geografía a lo largo de los años escolares. Así, tomando como referencia estos estudios, presentamos las tablas 2 y 3 que sistematizan el análisis de los mapas presentes en los libros de texto que realizamos en nuestra investigación a partir de las contribuciones teórico-metodológicas de Simielli (1999) y Duarte (2016).

\begin{tabular}{|c|c|c|c|c|c|c|}
\hline \multirow{2}{*}{ Niveles de actividades } & \multicolumn{3}{|c|}{$\begin{array}{c}\text { Conexiones: estudios de } \\
\text { geografía general y Brasil }\end{array}$} & \multicolumn{3}{c|}{ Geografía: espacio e identidad } \\
\cline { 2 - 7 } & $1^{\circ}$ Año & $2^{\circ}$ Año & $3^{\circ}$ Año & $1^{\circ}$ Año & $2^{\circ}$ Año & $3^{\circ}$ Año \\
\hline Localización y análisis & 49 & 34 & 42 & 50 & 47 & 53 \\
\hline $\begin{array}{c}\text { Localización y análisis / } \\
\text { Correlación }\end{array}$ & 6 & 17 & 15 & 18 & 27 & 3 \\
\hline $\begin{array}{c}\text { Localización y análisis / } \\
\text { Correlación / Síntesis }\end{array}$ & 0 & 2 & 1 & 0 & 0 & 0 \\
\hline
\end{tabular}

TABLA N ${ }^{\circ}$ 2: Mapas de libros de texto de acuerdo con los niveles de actividad propuestas por Simielli (1999), expresado según el número de ocurrencia. Fuente: Elaboración propia (2019)

\begin{tabular}{|c|c|c|c|c|c|c|}
\hline \multirow{2}{*}{$\begin{array}{c}\text { Niveles de } \\
\text { complejidad }\end{array}$} & \multicolumn{3}{|c|}{$\begin{array}{c}\text { Conexiones: estudios de geografía } \\
\text { general y Brasil }\end{array}$} & \multicolumn{3}{c|}{ Geografía: espacio e identidad } \\
\cline { 2 - 7 } & $1^{\circ}$ Año & $2^{\text {o }}$ Año & $3^{\circ}$ Año & $1^{\circ}$ Año & $2^{\circ}$ Año & $3^{\circ}$ Año \\
\hline Bajo & 46 & 26 & 42 & 57 & 56 & 52 \\
\hline Medio & 7 & 19 & 10 & 9 & 14 & 6 \\
\hline Alto & 2 & 8 & 6 & 2 & 4 & 1 \\
\hline
\end{tabular}

$\mathrm{TABLA} \mathrm{N}^{0} 3$ : El libro de texto asigna de acuerdo con los niveles de complejidad propuestos por Duarte (2016) expresado según el número de ocurrencia. Fuente: Elaboración propia (2019)

De los datos en la tabla 2, se observa que la ubicación y el análisis son las actividades más solicitadas para que los estudiantes aprendan a través de los mapas durante la enseñanza media (educación secundaria). En un momento en que el libro de texto se limita a requerir que los estudiantes, en su mayor parte, solo localicen los fenómenos, se hace evidente que el razonamiento más complejo de los estudiantes está poco movilizado para desarrollar el trabajo de geografía vinculado a los mapas. Se esperaba que a lo largo de los años escolares habría una mayor cantidad de mapas que requerían que los estudiantes correlacionaran y sintetizaran los fenómenos en el segundo año y, más tarde, más intensamente en el tercer año, el último año de secundaria. En un estudio comparado entre los currículos brasileño y español, Breda, García de la Vega y 
Straforini (2016) muestran el dominio de la identificación y localización de los lugares como actividad procedimental principal en relación a los contenidos cartográficos de la educación primaria.

Los tres niveles de actividad cartográfica propuestos por Simielli (1999) tenían la intención de resolver las brechas dejadas en el proceso de enseñanza-aprendizaje de la geografía, en particular, la falta de conocimiento de los contenidos cartográficos en la formación de los estudiantes. Para este autor, al dominar estos tres niveles, el estudiante podría comprender mejor su vida diaria, los aspectos que interfieren en sus vidas y contribuyen a la construcción de la ciudadanía.

Basado en la tabla 3, es interesante observar cómo se analizan los mapas desde la perspectiva de los estudios de Duarte (2016), que reconoce la importancia de que los estudiantes de la enseñanza media (educación secundaria) tengan la posibilidad de emplear y desarrollar un razonamiento más complejo a lo largo de sus antecedentes educativos y pueden usarlos para leer e interpretar los mapas presentes en los libros de texto de geografía. Nuevamente, lo que encontramos fue una estructura totalmente inversa de lo que esperan las teorías, es decir, los mapas más complejos presentes en el tercer año están en menor número en relación con los mapas de baja complejidad, esos presentes en números más significativo en el primer año de la secundaria.

A partir de estos datos podemos inferir que la presencia de mapas en los libros de texto de geografía de secundaria ofrece poca contribución al desarrollo de cogniciones más complejas, tomando como referencia la perspectiva de Morin (2000), quien destaca que los cuatro elementos del conocimiento pertinente serán importantes para la formación de pensamiento complejo.

Así es posible identificar el desajuste entre los estudios teóricos y metodológicos y su materialización en la producción de libros de texto disponibles para maestros y estudiantes. Por lo tanto, se considera importante enfatizar que el libro de texto es el material al que los maestros de geografía tienen un amplio acceso, y que a partir de él a menudo se elaboran las clases, la planificación del contenido y las evaluaciones. Además, el libro también tiene un carácter formativo para el profesor, ya que es el primer recurso al que recurre el profesor en el momento de la duda (Mota, 2015).

\section{CONSIDERACIONES FINALES}

Esta investigación nos permitió tener una visión general del lenguaje cartográfico en dos colecciones de libros de texto de geografía de secundaria, a partir del número total de mapas, de los diferentes tipos de cartografías que componen estos materiales, así como las propuestas y conceptos de presentar los mapas en libros con la perspectiva de la contribución al proceso de enseñanza-aprendizaje de la geografía. 
De este estudio pudimos entender que los niveles de actividad presentados por Simielli (1999), así como las preocupaciones expuestas por Duarte (2016), no fueron contextos tomados como referencia para la producción del lenguaje cartográfico presente en los libros de texto de geografía. Fue notable la poca preocupación de estos materiales al presentar una propuesta que contempla la cartografía más allá de la ilustración y que efectivamente contribuye al desarrollo del aprendizaje de los contenidos geográficos.

En cuanto a las colecciones, se puede observar que no hay diferencias notables entre sí, lo que nos permite desarrollar un análisis general, aunque solo sea un muestreo, de la linealidad existente en la elaboración de mapas para libros de texto de geografía de secundaria. Se esperaba que los materiales didácticos y el lenguaje cartográfico siguieran un camino constructivo para los estudiantes, es decir, que durante los años escolares los mapas presentes en los libros ganarían nuevas dimensiones, más información, más contenido y se convertirían efectivamente más complejos.

En este sentido, nos preocupan los resultados encontrados en este análisis, considerando que las proposiciones hechas por Simielli (1999) no son recientes. Por lo tanto, es urgente que los elementos de niveles de actividad y complejidad con el lenguaje cartográfico estén más presentes y sean coherentes en el contexto de la producción de libros de texto de geografía.

\section{BIBLIOGRAFÍA}

Almeida, R.D., 2007. Cartografia Escolar. São Paulo: Contexto.

Almeida, R.D., 2013. Do desenho ao mapa: iniciação cartográfica na escola. São Paulo: Contexto.

Brasil, 1998. Parâmetros Curriculares Nacionais: Geografia. Secretaria de Educação Fundamental. Brasília: SEF, 1998.

Brasil, 2017. PNLD 2018: Geografia - guia de livros didáticos - Ensino Médio. Brasília: Ministério da Educação/Secretária de Educação Básica, 2017.

Breda, T. V.; García de la Vega, A. y Straforini, A., 2016. Raciocínio espacial no ensino de Geografia. Uma análise da cartografia nos documentos curriculares da Espanha e Brasil. En Sebastíá, R. y Tonda, E. (Coords.): La investigación y la innovación en la enseñanza de la geografía, Alicante: Universidad de Alicante, pp. 899-913.

Duarte, R.G., 2016. Educação Geográfica, Cartografia Escolar e Pensamento Espacial no segundo segmento do ensino fundamental. Tese de Doutorado. São Paulo: USP. 
Fonseca, F.P., 2013. Cartografia. São Paulo: Melhoramentos.

García de la Vega, A. (2018). Análisis del currículo español: perspectiva desde la geografía y su didáctica. Revista Brasileira de Educação em Geografia, v. 8, n. 15 , pp. 5-38.

García de la Vega, A., 2019. Spatial Thinking Ability Acquisition through Geospatial Technologies for Lifelong Learning. En De Miguel González, R; Donert, K. y Koutsopoulos, K. (Eds.): Geospatial Technologies in Geography Education, Cham (SW): Springer, pp. 21-40.

Girardi, G., 2012. Mapas Alternativos e Educação Geográfica. Percursos, v. 13, n. 02, pp. 39-51.

Harley, J.B., 1991. A nova história da cartografia. O Correio da Unesco, v. 19, n. 8, pp. 4-9.

Lévy, J., 2008. Uma virada cartográfica? En Acselrad, H. (Ed.). Cartografias sociais e território. Rio de Janeiro: UFRJ/IPPUR, pp. 153-167.

Martinelli, M., 2017. Cartografia: reflexões acerca de uma caminhada. Revista Brasileira de Educação em Geografia, v. 7, n. 13, pp. 21-50.

Mota, H.G.S., 2015. O livro didático mediando a construção do conhecimento na formação continuada do professor de Geografia. Dissertação de Mestrado. Goiânia: UFG.

Morin, E., 2000. Os sete saberes necessários à educação do futuro. 2 ed. São Paulo: Cortez.

Oliveira, L., 1978. Estudo Metodológico e Cognitivo do Mapa. Tese de Livre Docência. São Paulo: IGEOG/USP, 1978.

Richter, D., 2011. O mapa mental no ensino de Geografia: concepções e propostas para o trabalho docente. São Paulo: Cultura Acadêmica.

Richter, D., 2017. A linguagem cartográfica no ensino em Geografia. Revista Brasileira de Educação em Geografia, v. 7, n. 13, pp. 277-300.

Santos, C., 2003. A Cartografia nos livros didáticos de Geografia: contrapontos de uma pesquisa. Revista Ciências Humanas, v. 9, n. 2, pp. 107-114.

Seemann, J., 2012. Subvertendo a Cartografia escolar no Brasil. Geografares, n. 12, pp. 138-174.

Simielli, M.E.R., 1999. Cartografia no ensino fundamental e médio. En Carlos, A.F.A.A, eds. Geografia em sala de aula. São Paulo: Contexto, pp. 92-108.

Simielli, M.E.R., 1986. O mapa como meio de comunicação: implicações no ensino de Geografia do $1^{\circ}$ grau. Tese de Doutorado. São Paulo: USP. 
Tonini, I.M.; Claudino, S. y Souto González, X.M., 2016. Manuais escolares de Geografia de Brasil, Espanha e Portugal: quais as inovações didáticas para o ensino de Geografia. En Sebastíá, R. y Tonda, E. (Coords.). La investigación y la innovación en la enseñanza de la geografía, Alicante: Universidad de Alicante, pp. 869-884. 